Unfallchirurg 2019·122:243-246

https://doi.org/10.1007/s00113-019-0603-2

Online publiziert: 21. Januar 2019

(c) Der/die Autor(en) 2019

\section{Redaktion}

P. Biberthaler, München

F. Gebhard, Ulm

\section{CrossMark}

Benjamin Lucas ${ }^{1}$. Dominik Brammen ${ }^{2}$. Wiebke Schirrmeister ${ }^{1}$ Jacob Aleyt ${ }^{3}$. Martin Kulla ${ }^{4}\left(\mathbb{D} \cdot\right.$ Rainer Röhrig $^{5} \cdot$ Felix Walcher $^{1}$

${ }^{1}$ Klinik für Unfallchirurgie, Universitätsklinikum Magdeburg, Magdeburg, Deutschland

${ }^{2}$ Klinik für Anästhesiologie und Intensivmedizin, Universitätsklinikum Magdeburg, Magdeburg, Deutschland

${ }^{3}$ TMF - Technologie- und Methodenplattform für die vernetzte medizinische Forschung e. V., Berlin, Deutschland

${ }^{4}$ Klinik für Anästhesie, Intensivmedizin, Notfallmedizin und Schmerztherapie, Bundeswehrankenhaus Ulm, Ulm, Deutschland

${ }^{5}$ Abteilung Medizinische Informatik, Department für Versorgungsforschung, Carl von Ossietzky

Universität Oldenburg, Oldenburg, Deutschland

\title{
Anforderungen an eine nachhaltige Standardisierung und Digitalisierung in der klinischen Notfall- und Akutmedizin
}

\section{Hintergrund}

Primäre Aufgabe der ärztlichen Dokumentation ist die Informationsweitergabe innerhalb des Behandlungsablaufs. Seit Einführung des $\$ 630 \mathrm{f}$ im Bürgerlichen Gesetzbuch (BGB) besteht eine gesetzliche Pflicht zur Dokumentation; damit hat der Patient ein Anrecht auf eine fachgerechte Dokumentation. Weiterhin sind die dokumentierten Daten für die Abrechnung, das Qualitätsmanagement, die Unternehmenssteuerung und die (Versorgungs-)Forschung unverzichtbar [17]. Diese Anforderungen gelten ebenso für die Dokumentation der Versorgung in einer Notaufnahme. Um einen Vergleich zwischen mehreren Standorten im Sinne eines Benchmarking zu ermöglichen, ist eine einheitliche Dokumentation notwendig. Diese Systematisierung und Standardisierung der Dokumentation wurden bereits vor über 50 Jahren gefordert [8]. Seit 1988 fordert das Sozialgesetzbuch V die Verbesserung von Qualität und Wirtschaftlichkeit im Gesundheitswesen durch elektronisch und maschinell verwertbare Kommunikation. In einem aktuellen Beschluss des Gemeinsamen Bundesausschusses (G-BA) über die Erstfassung zu einem gestuften System von Notfallstrukturen in Krankenhäusern [3] wird in $\$ 12$ nun ebenfalls gefordert, dass die Dokumentation in Notaufnahmen an Mindeststandards angelehnt sein muss. Die Deutsche Interdisziplinäre Vereinigung für Intensiv- und Notfallmedizin (DIVI) hat bereits 2010 mit dem Notaufnahmeprotokoll [15] einen Standard für die Dokumentation aller Patienten, vom fußläufigen Notaufnahmepatienten bis hin zu schwer verletzten [16] und schwer kranken Patienten jedweder Fachrichtung, definiert. Dieser liegt mit Stand Juli 2018 in der Version 2015.1 vor $[4,9]$.

Anlässlich eines gemeinsamen parlamentarischen Abends „Digitalisierung in der Notfallmedizin“ des Verbundforschungsprojekts „Verbesserung der Versorgungsforschung in der Akutmedizin durch Aufbau eines nationalen Notaufnahmeregisters" (AKTIN , BMBF gefördert, Förderkennzeichen: 01KX1319) und der Technologie- und Methodenplattform für die vernetzte medizinische Forschung e. V. (TMF) in Berlin wurden
5 zentrale Forderungen formuliert und mit Vertretern der Politik diskutiert.

\section{Forderung 1 - einheitlicher Dokumentationsstandard}

Als eine der zentralen Forderungen wurde die „... Einführung eines einheitliche(r) Dokumentationsstandard in der Notfallmedizin ..." aufgestellt (- Infobox 1) [7].

Durch die Einführung von elektronischen Notaufnahmedokumentationssystemen ist grundsätzlich die Wiederverwendbarkeit von einmal dokumentierten Daten möglich. Um den Aufwand für den Abgleich von Datendefinitionen zwischen den unterschiedlichen IT-Systemen in Vertragsarztsektor, Rettungsdienst, Notaufnahmen und Krankenhäusern auf das Mindestmaß zu begrenzen, müssen die Daten in einer harmonisierten und standardisierten Form nach einer einheitlichen Definition erhoben werden. Diese Datendefinitionen ermöglichen die Abbildung auf ein kontrolliertes medizinisches Vokabular für die Verwendung unter oben genannten Versorgern. Das medizinische Vokabular 


\section{Infobox 1 Fünfpunktepro- gramm zu Standardisierung und Digitalisierung in der klinischen Notfall- und Akutmedizin}

- Verpflichtender, national einheitlicher Dokumentationsstandard in der Notfallversorgung

- Nutzung international etablierter, offener Kommunikationsstandards (Health Level 7 - Clinical Document Architecture, Health Level 7 - Fast Healthcare Interoperability Resources, Logical Observation Identifiers Names and Codes, Systematisierte Nomenklatur der Medizin Clinical Terms, Integrating the Healthcare Enterprise)

- Schaffung einer nationalen Stelle zur Qualitätssicherung in der Notfallmedizin

- Verstetigung der Infrastruktur für das Notaufnahmeregister

- Vergütungsstruktur als Anreiz für die Einhaltung einer Mindestdokumentation

muss von einer übergeordneten nationalen Stelle kontrolliert und gepflegt werden. International werden solche Stellen "value set authorities" genannt. Eine solche Institution sollte aus Sicht der Autoren in der öffentlichen Hand liegen, wie z.B. das Deutsche Institut für Medizinische Dokumentation und Information (DIMDI), welches schon verschiedene Kataloge wie die deutsche Version der internationalen statistischen Klassifikation der Krankheiten (ICD), die Identifikationsnummer für Diagnosen (Alpha-ID) oder den Operationen- und Prozedurenschlüssel (OPS) pflegt. Das DIMDI oder die zu schaffende Institution sollte diese Aufgabe in Zusammenarbeit mit den Domänenexperten aus Fachgesellschaften, Industrie und Standardisierungsorganisationen übernehmen.

\section{Forderung 2 - Nutzung von Interoperabilitätsstandards}

Um die harmonisierten und standardisierten Daten zwischen den unterschiedlichen IT-Systemen der an der Notfallversorgung Beteiligten auszutauschen, sind einheitliche Kommunikationsstandards notwendig. Dazu bieten sich international bereits etablierte Kommunikationsstandards an, die bereits größtenteils in Deutschland im Einsatz sind. Schnittstellen benötigen dabei ei- ne zweistufige Standardisierung. Durch die syntaktische Standardisierung wird in dem Kommunikationsstandard definiert, an welcher Stelle Informationen in welcher Art und Weise übermittelt werden. Für medizinische Dokumente bietet sich die Nutzung des Kommunikationsstandards Health Level 7 - Clinical Document Architecture (HL7-CDA) [5] an. Neben syntaktischen Kommunikationsstandards müssen zur interoperablen Datenweiternutzung semantische Standards verwendet werden. Sie definieren, welche Informationen übermittelt werden. Dazu werden dem kontrollierten medizinischen Vokabular durch die „value set authority codes“ zugeordnet, die die automatisierte elektronische Verarbeitung der Informationen erst ermöglichen. Beispielsweise wird derzeit durch das DIVI-Codesystem 41 die Transportmethode des Patienten hinterlegt. Der Code „1“ steht hierbei für den Krankentransportwagen [6]. Damit ist die eindeutige Zuordnung der Transportmethode unabhängig von $\mathrm{Ab}$ kürzungen, synonymen Begriffen oder Multilingualität zwischen verschiedenen Systemen möglich. International und national bereits verwendete Standards an dieser Stelle sind die ICD-10 zur Diagnosenkodierung und der OPS zur Kodierung von Maßnahmen. Zunehmende Verbreitung findet zur Kodierung von Laborinformationen das kostenfrei nutzbare Kodesystem „Logical Observation Identifiers Names and Codes" (LOINC) [10]. Für die Kodierung von klinischen Inhalten, die in ICD-10, OPS und LOINC nicht abgedeckt sind, steht die international verwendete Ontologie Systematisierte Nomenklatur der Medizin Clinical Terms (SNOMED CT) zur Verfügung [13]. Diese umfasst aktuell knapp 350.000 Konzepte, die über mehr als 1 Mio. Beziehungen miteinander verbunden sind und so die Daten einfacher auswertbar macht. Die Konzepte sind international standardisiert und werden national mit einem oder mehreren (synonymen) Begriffen (,terms“) bezeichnet.

Im Rahmen des AKTIN-Projektes wurden bereits Teile des Notaufnahmeprotokolls als HL7-CDA modelliert. Das für jeden Fall notwendige Basismodul wurde, wie das für die Schwerverletz- tenversorgung und Übermittlung in das TraumaRegister DGU $^{\circledR}$ [14] notwendige Traumamodul, bereits mit HL7CDA und unter Nutzung von ICD10, OPS, LOINC und SNOMED CT umgesetzt. Gerade die Nutzung von SNOMED CT ist für die einheitliche Semantik unverzichtbar, gestaltet sich jedoch aus Lizenzgründen in Deutschland schwierig. Für die flächendeckende Verwendung ist der Erwerb einer nationalen Lizenz vergleichbar z. B. der ICD10 notwendig. Viele unserer Nachbarstaaten wie z.B. Niederlande, Belgien und Großbritannien verfügen bereits über diese Terminologie. Hier ist das nunmehr dringende Handeln der Bundespolitik bzw. des Bundesministeriums für Gesundheit sowie der Deutschen Krankenhausgesellschaft, des GB-A und der Kassenärztlichen Bundesvereinigung gefragt, um eine einheitliche Semantik und damit weiterführend die gesetzlich geforderte Qualitätssicherung in der Notfallmedizin zu ermöglichen. Nationale Alleingänge machen weder aus Sicht der Industrie noch aus Sicht von Patienten (internationaler Austausch von Behandlungsdaten) oder der Forscher Sinn bzw. werden Deutschland schon kurz- bis mittelfristig von der internationalen Entwicklung abhängen.

\section{Forderung 3 - Qualitäts- sicherung in der klinischen Notfall- und Akutmedizin}

Die Schaffung einer nationalen Stelle zur Qualitätssicherung in der Notfallmedizin ist notwendig, um ein bundesweit einheitliches Qualitätsmanagement $\mathrm{zu}$ etablieren. Hierzu erarbeitet das Projekt AKTIN die Grundlagen für ein nationales Notaufnahmeregister. Als Basis der Datenstruktur wird hier das Basismodul des DIVI-Notaufnahmeprotokoll V2015.1 verwendet [9]. Auf Grundlage der Fortschritte in der Standardisierung und Digitalisierung in der Notfallmedizin wird mithilfe des Notaufnahmeregisters eine bundesweit einheitliche Infrastruktur für Qualitätsmanagement, Infektions-Surveillance und Versorgungsforschung für die Notaufnahmebehandlung entwickelt. Derzeit kooperieren 15 Modellkliniken im AK- 
TIN-Projekt, die den standardisierten Export des Notaufnahmeprotokolls aus den individuellen elektronischen Notaufnahmeinformationssystemen in das Notaufnahmeregister im Hinblick auf die Realisierbarkeit der IT-Infrastruktur testet. Neben der Sekundärnutzung im Notaufnahmeregister bietet das Notaufnahmeprotokoll der DIVI mit dem Traumamodul einen Dokumentationsstandard für Schockraumpatienten, der alle Daten der Schwerverletztenversorgung im TraumaRegister $\mathrm{DGU}^{\circledR}$ für den Behandlungsabschnitt im Schockraum umfasst $[11,16]$. Auch hier ist mithilfe einer interoperablen elektronischen Implementation in den Klinikinformationssystemen bzw. Notaufnahmeinformationssystemen eine automatisierte Übertragung in das TraumaRegister $\mathrm{DGU}^{\circledR}$ geplant, um den retrospektiven, webbasierten Dokumentationsaufwand und damit die Redundanz entscheidend $\mathrm{zu}$ verringern [11]. Auf Basis der hier geschaffenen Grundlage ist es erforderlich, eine Ausweitung der Infrastruktur auf die nationale Ebene zu erarbeiten und damit eine nationale Qualitätssicherungsstelle für die klinische Akut- und Notfallmedizin zu schaffen.

\section{Forderungen 4 und 5 - Verstetigung und Vergütung}

Die technische Machbarkeit eines nationalen Notaufnahmeregisters, welches automatisch mit Daten aus der Routineversorgung gespeist wird, konnte gezeigt werden [2]. Durch dezentrale Datenhaltung im Organisationsbereich der Notaufnahmen sind die Einhaltung des Datenschutzes und der Dateneignerschaft sichergestellt [1]. Die Nutzung dieser Daten aus dem Notaufnahmeregister zu Zwecken der Versorgungsforschung, Qualitätssicherung und Surveillance wird zurzeit evaluiert. Aktuell ist ein monatliches Berichtswesen der 15 Modellkliniken etabliert; das einrichtungsübergreifende Benchmarking befindet sich derzeit im Aufbau. Für die Versorgungsforschung stehen bereits erste Daten zur Verfügung. Im nächsten Schritt ist die Verstetigung der hier geschaffenen Infrastruktur notwendig, damit das Notaufnahmeregister

Unfallchirurg 2019 · 122:243-246 https://doi.org/10.1007/s00113-019-0603-2

(c) Der/die Autor(en) 2019

B. Lucas · D. Brammen · W. Schirrmeister · J. Aleyt · M. Kulla · R. Röhrig · F. Walcher

Anforderungen an eine nachhaltige Standardisierung und Digitalisierung in der klinischen Notfall- und Akutmedizin

\section{Zusammenfassung}

Standardisierung und Digitalisierung der Dokumentation in der Medizin schreiten zunehmend voran. In dem Beschluss des Gemeinsamen Bundesauschusses zu einem gestuften System von Notfallstrukturen in Krankenhäusern sowie in dem Gutachten des Sachverständigenrates zur Entwicklung im Gesundheitswesen Bedarfsgerechte Steuerung der Gesundheitsversorgung wird die Aktualität des Themas mit konkreter Nennung der Zukunftsaufgaben unterstrichen. Die Sektion Notaufnahmeprotokoll der Deutschen Interdisziplinäre Vereinigung für Intensivund Notfallmedizin e. V. (DIVI) arbeitet seit Jahren an diesem Thema und hat wiederholt über den Fortschritt des Themas in der klinischen Notfallversorgung berichtet. Standardisierung und Digitalisierung stellen neben der Möglichkeit für Benchmarking die Grundlage für eine standortübergreifende Versorgungsforschung dar. Mit der Digitalisierung wird die Sekundärnutzung primärer klinischer Daten möglich. Unnötige Redundanzen bei der Erhebung und der Datenübermittlung in z. B. Register werden reduziert.

Schlüsselwörter

Digitalisierung $\cdot$ Standardisierung .

Notaufnahme - Versorgungsforschung .

Notaufnahmeregister

\section{Requirements for a sustainable standardization and digitalization in clinical emergency and acute medicine}

Abstract

Digitalization and standardization of documentation in medicine are increasingly progressing. In the decision of the Federal Joint Committee (G-BA) for a staged system of emergency structures in hospitals and in the report of the expert committee for development in the healthcare system on needs-oriented guidance of healthcare, the actuality of the topic is underlined with concrete naming of future tasks. The section on emergency admission protocols of the German Interdisciplinary Association of Intensive and Emergency Care Medicine (DIVI) has been working for years on this topic and has repeatedly reported on the progress of the topic in clinical emergency care. Standardization and digitalization represent the foundation for health services research spread across locations as well as the possibility for benchmarking. Digitalization makes the secondary use of primary clinical routine data possible. Digitalization decreases redundancies of data transmission by avoiding manual data input in, for example registers.

\section{Keywords}

Digitalization · Standardization · Emergency department . Health services research . Emergency department registry auf alle Notaufnahmebehandlungen in Deutschland, ähnlich dem TraumaRegister $\mathrm{DGU}^{\circledR}$ für die Schockraumbehandlung von verunfallten Patienten, ausgeweitet werden kann. Der Sachverständigenrat zur Begutachtung der Entwicklung im Gesundheitswesen fordert in seinem aktuellen Gutachten aus dem Jahr 2018 zur Bedarfsgerechte[n] Steuerung der Gesundheitsversorgung ebenfalls den Ausbau des Notaufnahmeregisters im AKTIN-Projekt sowie die Entwicklung von Qualitätsindikatoren für die Versorgung in Notaufnahmen [12].
Hierfür ist ebenso die Schaffung einer Vergütungsstruktur notwendig, um den Mehraufwand für die Digitalisierung und Standardisierung aufzuwiegen. Hier ist insbesondere ein zusätzliches Anreizsystem notwendig, da eine ungleiche Verteilung von Aufwand und Nutzen beim Dokumentierenden und dem Informationsempfänger besteht. Eine standardkonforme Mindestdokumentation sollte, nach dem Vorbild des Meaningfuluse-Programms in den USA, auch in Deutschland besonders vergütet werden. Damit werden die Umsetzung des gesetz- 
lich geforderten Mindeststandards der Dokumentation gefördert und die $\mathrm{Be}$ reitstellung entsprechender Daten für das Benchmarking und die Qualitätssicherung verbessert.

\section{Ausblick}

Die Einführung eines einheitlichen Dokumentationsstandards und der Einsatz von Interoperabilitätsstandards zur Qualitätssicherung der klinischen Notfall- und Akutmedizin wurde durch das AKTIN-Projekt (www.aktin.org) zum Aufbau eines Notaufnahmeregisters exemplarisch umgesetzt. Aktuell können weitere interessierte Kliniken, bei Vorhandensein der HL7-CDA-Exportschnittstelle, am Notaufnahmeregister teilnehmen, um die Verbreitung in Deutschland voranzubringen. Parallel dazu arbeitet die Sektion Notaufnahmeprotokoll an dem Abgleich und der Integration der in Deutschland etablierten Registerdatensätze wie z.B. TraumaRegister $\mathrm{DGU}^{\circledR}$, Reanimationsregister oder Schlaganfallregister für das nächste Datensatz-Update 2020.

Zur Umsetzung der erhobenen Forderungen ist die aktive Mitwirkung der Politik, des G-BA, der Deutschen Krankenhausgesellschaft (DKG) und des Spitzenverbandes Bund der Krankenkassen (GKV-Spitzenverband) zwingend erforderlich.

\section{Korrespondenzadresse}

Dr. Benjamin Lucas
Klinik für Unfallchirurgie,
Universitätsklinikum
Magdeburg
Leipziger Straße 44,
39120 Magdeburg,
Deutschland
benjamin.lucas@
med.ovgu.de

Interessenkonflikt. B. Lucas, D. Brammen, W. Schirrmeister, M. Kulla, R. Röhrig und F. Walcher wirken im Verbundprojekt „Verbesserung der Versorgungsforschung in der Akutmedizin durch den Aufbau eines nationalen Notaufnahmeregisters" (AKTIN, BMBFgefördert, 01KX1319) mit. F. Walcher ist Sprecher, M. Kulla ist Stellvertreter und D. Brammen Schriftführer der Sektion Notaufnahmeprotokoll der DIVI. B. Lucas und R. Röhrig sind Mitglieder der Sektion Notaufnahmeprotokoll. M. Kulla erhält Fördermittel vom Bundesministerium der Verteidigung (SoFo 34K3-17 1515 DigiPen). W. Schirrmeister, M. Kulla,
R. Röhrig und F. Walcher wirken im Projekt „ENQuIRE - Evaluierung der Qualitätsindikatoren von Notaufnahmen auf Outcome-Relevanz für den Patienten" (VSF1_2017-020) mit, welches vom G-BA gefördert wird. J. Aleyt war während der Erarbeitung dieses Papers als wissenschaftlicher Mitarbeiter bei der TMF - Technologie- und Methodenplattform für die vernetzte medizinische Forschung e. V. beschäftigt. Das Projekt "Verbesserung der Versorgungsforschung in der Akutmedizin in Deutschland durch den Aufbau eines Nationalen Notaufnahmeregisters" (AKTIN) ist Mitglied der TMF.

Open Access. Dieser Artikel wird unter der Creative Commons Namensnennung 4.0 International Lizenz (http://creativecommons.org/licenses/by/4.0/deed. de) veröffentlicht, welche die Nutzung, Vervielfältigung, Bearbeitung, Verbreitung und Wiedergabe in jeglichem Medium und Format erlaubt, sofern Sie den/die ursprünglichen Autor(en) und die Quelle ordnungsgemäßnennen, einen Link zur Creative Commons Lizenz beifügen und angeben, ob Änderungen vorgenommen wurden.

\section{Literatur}

1. Ahlbrandt J, Brammen D, Majeed RW et al (2014) Balancing the need for big data and patient data privacy-an IT infrastructure for a decentralized emergency care research database. Stud Health Technol Inform 205:750-754

2. Brammen D, Dewenter $H$, Thiemann V et al (2017) Disseminating a standard for medical records in emergency departments among different software vendors using HL7 CDA. Stud Health Technol Inform 243:132-136

3. Gemeinsamer Bundesausschuss (2018) Beschluss des Gemeinsamen Bundesausschusses über die Erstfassung der Regelungen zu einem gestuften System von Notfallstrukturen in Krankenhäusern gemäß§136c Absatz 4SGBV

4. Greiner F, Brammen D, Kulla Met al (2017) Standardisierte Erhebung von Vorstellungsgründen in der Notaufnahme. Med Klin Intensivmed Notfallmed. https://doi.org/10.1007/s00063-017-0286-9

5. HL7 CDA (2018) Clinical document architecture: HL7 Deutschland e. V. http://hl7.de/themen/hl7cda-clinical-document-architecture/. Zugegriffen:4. Juli 2018

6. HL7 Deutschland DIVI Codesystem 41. http://wiki. hl7.de/index.php?title=1.2.276.0.76.11.41/static2015-11-17T000000. Zugegriffen:04. Juli 2018

7. Interview: „Wir brauchen einen einheitlichen Dokumentationsstandard in der Notfallversorgung". https://idw-online.de/de/news692951. Zugegriffen:07. Mai 2018

8. Koller S, Überla K (1966) Die Verwendung elektronischer Rechenanlagen in der Medizin II. Fortschr Med 84:279-282

9. Kulla M, Brammen D, Greiner F et al (2016) Vom Protokoll zum Register - Entwicklungen für ein bundesweites Qualitätsmanagement in deutschen Notaufnahmen. DIVI 7:12-20. https:// doi.org/10.3238/DIVI.2016.0012-0020

10. LOINC (2018) The freely available standard for identifying health measurements, observations, and documents. https://loinc.org/. Zugegriffen: 4 Juli 2018

11. Ruchholtz S, Siebert H (2012) Easier documentation for certified trauma centers oft (corrected) the German Society for accident surgery (corrected).
Unfallchirurg 115:465. https://doi.org/10.1007/ s00113-012-2221-0

12. Sachverständigenrat zur Begutachtung der Entwicklung im Gesundheitswesen (2018) Bedarfsgerechte Steuerung der Gesundheitsversorgung. https://www.svr-gesundheit.de/index. php?id=606. Zugegriffen: 12 . Juli 2018

13. SNOMED International SNOMED CT (2018) https:// www.snomed.org/snomed-ct/what-is-snomedct.Zugegriffen: 4. Juli 2018

14. TraumaRegister DGU ${ }^{\circledR}$ (2014) 20 years TraumaRegister DGU ${ }^{\circledR}$ : development, aims and structure. Injury 45(Suppl 3):S6-S13. https://doi.org/10. 1016/j.injury.2014.08.011

15. Walcher F, Kulla M (2011) Kerndatensatz "Notaufnahme". Dtsch Arztebl 108:A626-A628

16. Walcher F, Kulla M, Klinger $S$ et al (2012) Standardisierte Dokumentation im Schockraum mit dem Kerndatensatz "Notaufnahme" der DIVI. Unfallchirurg 115:457-464. https://doi.org/10. 1007/s00113-012-2220-1

17. Wiesenauer M, Johner C, Röhrig R(2012) Secondary use of clinical data in healthcare providers - An overview on research, regulatory and ethical requirements. Stud Health Technol Inform 180:614-618. https://doi.org/10.3233/978-161499-101-4-614 